\title{
A comparison of old and young rats' performance on a test of nonmatching-to-sample: An analysis of age-related encoding and memory deficits
}

\author{
SYLVAIN GAGNON \\ Rotman Research Institute, Baycrest Centre for Geriatric Care, North York, Ontario, Canada \\ and \\ GORDON WINOCUR \\ Rotman Research Institute, Baycrest Centre for Geriatric Care, North York, Ontario, Canada \\ and Trent University, Peterborough, Ontario, Canada
}

\begin{abstract}
Using a multiple probe testing procedure (Roitblat, 1980), young and old rats were tested on a nonmatching-to-sample (NMTS) task in a three-arm maze. Subjects were forced to visit two different arms successively and, after variable delays, were required to choose the nonmatching arm. Incorrect responses on an initial probe run were followed by a second probe run. On no-delay trials, old rats performed poorly on first probe runs but they improved significantly on second probe runs. At longer delays, old rats exhibited faster rates of decline than did young rats. The results indicated that old rats suffered a decline in their ability to encode new information and rapid memory loss, and that the two deficits combined to adversely affect overall performance. Results were related to neuropsychological evidence that implicated prefrontal and hippocampal dysfunction in the types of age-related deficits observed in the present study.
\end{abstract}

Several studies have compared learning and memory performance in old and young animals on tests of matching (MTS) and nonmatching-to-sample (NMTS). Such tests comprise pairs of trials in which the usual procedure is to present subjects with a sample stimulus during an initial study run. After a delay, a comparison run follows in which the same stimulus is introduced along with a novel stimulus. The animal is rewarded for selecting the familiar stimulus in MTS and the unfamiliar stimulus in NMTS. The evidence indicates that aged rats and monkeys are reliably impaired in learning both tests and that age differences increase with the length of the interval between the study and comparison runs (Dunnet, Evenden, \& Iversen, 1988; Dunnet, Martel, \& Iversen, 1990; Pontecorvo, Clissold, \& Conti, 1988; Walker et al., 1988; Winocur, 1992a; but see Aggleton, Blindt, \& Candy, 1989).

The delay-dependent deficits of old animals on tests of NMTS and MTS are consistent with similar findings

The present research was supported by a grant from the Natural Sciences and Research Council of Canada (A-8181) to G.W. and an NSERC postdoctoral fellowship to S.G. The authors wish to thank Sue Di Francesco and John Zomer for their technical assistance. This paper was completed while G.W. was a visiting professor at the Hebrew University, Jerusalem. Thanks are expressed to Shlomo Bentin, his lab colleagues, and the Department of Psychology for their hospitality and use of their facilities. S. Gagnon is now at the Département de Psychologie, Pavillon Michel-Sarrazin, Université du Québec à Trois-Rivières, Trois-Rivières, C. P. 500, QC, Canada G9A 5H7 (e-mail: gagnons@) neptune.uqtr.uquebec.ca). based on tests of delayed alternation (Winocur, 1986; Zornetzer, Thompson, \& Rogers, 1982) and conditional associative learning (Winocur, 1992b), in which successful performance also depends on the animal's ability to recall trial-specific information. These results have been attributed to an age-related failure to retain acquired information (Winocur, 1992b; Zornetzer et al., 1982). While there is considerable evidence to support this view, the question that has not been satisfactorily addressed is the extent to which rapid forgetting in these tests is related to a deficit at an earlier stage in the learning process. Information that is improperly encoded at the time of presentation will be poorly learned and will be subject to rapid decay, regardless of the animal's ability to store learned information. Gallagher and Pelleymounter (1988) made a similar observation in their analysis of age-related deficits on spatial memory tasks. In describing the nature of such deficits, they pointed out that impaired performance could result from impoverished processing of spatial information and/or a decline in spatial memory function.

There has been little attempt to dissociate encoding and memory deficits in old animals and assess their respective contributions to overall performance. One reason for lack of progress in this area is that the tests of learning and memory that are commonly used to measure age differences in animals do not typically yield separate measures of underlying cognitive processes. A spatial test of MTS developed by Roitblat and his col- 
leagues to study the interactive influences of encoding and memory processes in young adult rats may prove useful in this regard (Adams-Pepper, Gagnon, Fore, \& Roitblat, 1992; Roitblat, 1980; Roitblat \& Harley, 1988; Roitblat, Harley, \& Helweg, 1989). This task makes use of a multiple probe technique to provide independent measures of encoding errors on the one hand, and errors resulting from memory decay on the other.

In Roitblat's original test, which was conducted in a three-arm starburst maze, rats were forced to enter one of the arms during a single study run. After a delay, rats received a comparison run (first probe) in which they were free to enter any of the three arms. When rats failed to select the sample arm, they were given a second probe run in which the erroneously entered arm was blocked. Correct responses on the first probe run indicated that information related to the sample stimuli had been encoded effectively and recalled. At short delay intervals, incorrect responses on the first probes were related to a deficit in encoding the sample arm. Inserting longer delays between the sample and probe runs provided an opportunity to assess memory loss. As expected, Roitblat and Harley (1988) observed that when the delay between the study and probe runs was increased, performance decreased accordingly.

The second probe run (following an error on the first probe) allowed a more finely grained assessment of processes involved in performing the MTS task. Improved performance on the second probe run at short and longer delays was seen as an indication that some relevant information had been processed and was accessible, and that first probe incorrect responses were caused by a failure to encode relevant information. Poor performance on second probe runs following a short delay would indicate that relatively little information about the sample was encoded during the study trials. A further decline in performance after an extended delay would raise the possibility that animals were also suffering memory loss.

At relatively long delays, a wrong response on the second probes could be seen as reflecting some combination of encoding and retention deficits. If one assumes that over the course of the trial (especially on the sample runs), each arm acquires some value with respect to correctness, it is reasonable to expect that on the first probe runs, rats would most likely select the arm that has the highest value. On the second probe, where rats are prevented from choosing the same arm again, they should select the arm that has acquired the second highest value. The combined influence of memory loss and encoding failure can be determined by comparing the decline in performance of the first probe (short- vs. long-delay trials) to the decline of performance on second probe runs (short- vs. long-delay trials). Faster decay on the second probe runs would imply that memory loss interacts with level of encoding because the stimulus information for second probe runs (following an error) would be more poorly encoded than for first probe runs in which rats responded correctly. A similar decay function for first and second probe runs would indicate that en- coding and memory loss are two independent processes that are affected by different variables.

In the present experiment, Roitblat's multiple probe technique was used to compare old and young rats on a test of NMTS in which the delay between study and probe runs was varied. An NMTS task was preferred because rats typically learn NMTS tasks faster than the MTS variety and because, particularly in older rats, variation in performance is usually less on NMTS tasks (Dunnett et al., 1988). The primary purpose was to assess the extent to which differences in encoding and storage abilities combine to account for age differences in performance on this task.

In another departure from Roitblat's original paradigm, on each trial we allowed rats two study runs in which they were forced to enter a different arm on each run. After the second study run, the rats were administered a probe run in which they were free to choose any of the arms, and a second probe run if an error had been made on the first probe. The interval between the second study run and the first probe run was varied. A response was reinforced when the rat chose the arm that it had not visited in the study trials (NMTS). This procedure has the advantage of providing an additional measure of memory loss at short and longer delays. Because rats are presented with two sample arms successively, a greater tendency to make first probe errors to the first sample arm than to the second sample arm would be consistent with a memory decay interpretation.

While several outcomes were possible, a reasonable expectation was that old rats would experience failures of both encoding and retention as part of a general deterioration of cognitive function. An encoding deficit would take the form of a large number of errors on first probe runs at short delays. Faster memory decay would be reflected in a decline in performance on first and second probe runs that corresponded to the length of the retention interval. If encoding and memory deficits interact with each other, greater loss would be expected on second probes than on first probes at long delays.

\section{METHOD}

\section{Subjects}

Ten old ( 24 months) and 10 young ( 4 months) rats of the Long Evans strain were obtained from the Trent University Animal Breeding Center. Two weeks before the beginning of the experiment, the rats were housed individually in standard wire mesh cages and handled daily. One week later, the rats were subjected to a food deprivation schedule and were maintained at $85 \%$ of their free-feeding body weight for the duration of the experiment. Rats were kept under a 9:15-h light:dark regimen and had free access to water at all times. The old rats were examined regularly by a veterinarian who certified that they were healthy and able to participate in the experiment.

\section{Apparatus}

The apparatus, which was made of wood and painted flat gray, consisted of an elevated three-arm starburst maze with a stem and a startbox. Each arm was $64 \mathrm{~cm}$ long $\times 17 \mathrm{~cm}$ wide; the startbox. was $20.5 \times 17 \mathrm{~cm}$; and the stem was $46 \times 17 \mathrm{~cm}$. The startbox and 
the three goal arms were separated from the stem by guillotine doors $(17.9 \mathrm{~cm}$ high $\times 16.4 \mathrm{~cm}$ wide). A recessed food cup, $3.5 \mathrm{~cm}$ in diameter, was located at the end of each goal arm. The startbox ( $17 \mathrm{~cm}$ high $\times 20.3 \mathrm{~cm}$ long $\times 17 \mathrm{~cm}$ wide) was covered by a Plexiglas top in which several $5-\mathrm{mm}$ holes were drilled. Wooden legs, $30 \mathrm{~cm}$ in height, supported the three goal arms and the stem.

The maze was placed on a table $(100 \times 60 \mathrm{~cm})$ in a quiet room $(2.8 \times 2.8 \mathrm{~m})$. Four overhead rectangular lights (each containing two $40-\mathrm{W}$ fluorescent bulbs) provided room illumination. There was another table $(91.5 \times 152 \mathrm{~cm})$ against one wall, on which the rats' cages, along with miscellaneous items, were kept. A cork board $(60.5 \times 60.5 \mathrm{~cm})$ hung on the wall behind the maze. During training and testing, the experimenter stood on the right of the maze.

\section{Procedure}

NMTS shaping and training. Rats were familiarized with the maze over a 7-day period. In the first 3 days, bits of Froot Loops (a commercial presweetened cereal) were scattered throughout the maze. Subjects were placed individually in the startbox, the Plexiglas cover was placed over the startbox, and the guillotine door was raised. Rats were allowed to walk freely in the stem and the three arms to eat the Froot Loops. A shaping session ended when all the Froot Loops were eaten or after $15 \mathrm{~min}$, whichever came first. On Days 4 and 5, Froot Loops were placed only in the three food cups and subjects had $15 \mathrm{~min}$ to eat them. On Days 6-8, one Froot Loop was placed in each food cup, the guillotine door of the startbox was raised, and the rat was allowed to visit the three goal arms and eat the food. After consumption of the Froot Loops, the rat was returned to the startbox, and the procedure was repeated for a total of five trials each day.

NMTS training began on Day 9. In this phase, rats were presented with the nonmatching-to-sample rule. A complete trial consisted of two forced-choice study runs followed by either one or two probe runs. On the first forced-study run, the rat was placed in the startbox, the cover was flipped down, and the guillotine door was raised. Only one arm was baited and open (the guillotine doors of the other two goal arms were lowered). As soon as the rat entered the open arm (i.e., four paws were in the arm), the guillotine door was lowered and the rat was allowed to eat the Froot Loops. After the rat ate the food, it was returned to the startbox for the second forced-study run. The procedure for the second study run was identical to that for the first study run, except that a different goal arm was baited and open.

After eating the food in the second forced-study arm, the rat was returned to the startbox and was administered the first free probe run. A delay of about $3 \mathrm{sec}$ separated the second study run and the probe run. On the probe run, the rat had to select, from three goal arms, the goal arm that it had not visited on the two study runs. The guillotine door of the startbox was raised and the rat was allowed to visit one of the three goal arms. As soon as the rat entered an arm (all four paws in the arm), the guillotine door was lowered. If the selection was correct, the rat was allowed to eat the food reward and was returned to its home cage for a $30-\mathrm{sec}$ intertrial interval (ITI). If the rat made an error, it was immediately returned to the startbox. The guillotine door of the erroneously chosen goal arm was lowered, and the rat now had to choose between the two remaining goal arms. If the choice on the second probe run was correct, the rat was allowed to eat the food and was returned to its home cage for a $30-\mathrm{sec}$ ITI. If the choice on the second probe run was incorrect, the rat was not reinforced and was returned immediately to its home cage to spend the ITI. Rats were given 10 trials a day until they reached a criterion of eight correct first probe responses on 4 consecutive sessions or after 20 sessions of training, whichever came first.

NMTS with delays. In this condition, which began the day after the last session in the zero-delay condition, a delay interval was introduced between the second forced-choice study run and the first free probe run. After the second study run, the rat was returned to the startbox to await the first probe trial. Rats received a total of 10 daily sessions with delays. For 5 sessions, the second study run-first probe run delay was $20 \mathrm{sec}$, and in the other 5 sessions, the delay was $90 \mathrm{sec}$. The delay for each session was determined by a prerandomized schedule, except that in the first session the delay was always $20 \mathrm{sec}$. At the end of the delay condition, each rat had received 120 trials ( 10 sessions $\times 12$ trials). For this phase of testing, the ITI was increased to $60 \mathrm{sec}$ to reduce possible interfering effects from the preceding trial.

\section{Statistical Analyses}

Analyses of variance (ANOVA) for a mixed design were computed to assess age differences across the three delays. The effect of delay was assessed by comparing the average number of successful probes over the last five training sessions (3-sec-delay trials) with the average number of successful probes over the five sessions with 20 -sec-delay trials, and the five sessions with 90 -secdelay trials. An age group $\times$ delay ANOVA with repeated measure on the last factor was computed for first- and second-probe performance. In the presence of a significant interaction, the ANOVA was followed by an analysis of simple main effects. If significant, these analyses were then followed by a post hoc mutiple comparison test (Tukey, HSD) to examine differences across the three delays within each group. Type I error was kept at .05 experimentwise.

In order to determine whether first- and second-probe performance significantly exceeded chance level, one-sample $t$ tests were performed. In the case of first-probe performance, the group mean was compared with a population average of $33 \%$, the probability of selecting the nonmatching arm among the three maze arms by chance being .33. For second-probe performance, a $50 \%$ population average was used, because only two arms could be probed and the probability of selecting the nonmatching arm by chance was increased to .5 . Once again, the average number of successful probes over the five sessions of each delay condition was used to determine whether the rats' performance was significantly superior to chance.

\section{RESULTS}

There were clear age differences between old and young rats in learning the NMTS task. Young rats required an average of 150 trials to reach the criterion of $80 \%$ first-probe accuracy on 4 consecutive sessions. In contrast, none of the old rats reached that criterion in the 20 training sessions.

Although the old rats failed to reach the criterial level of performance, there was evidence of substantial learning. In the last five training sessions, their average performance was $61 \%$ correct on first-probe trials, which is significantly higher than the $33 \%$ chance accuracy $[t(10)=$ $22.5, p \leq .0001]$. Old rats' first-probe accuracy rate improved from $37 \%$ (Day 1) to $61 \%$ (Day 20). Second-probe accuracy scores provide additional evidence of learning. Day 1 second-probe performance was at chance level, whereas on Day 20 , old rats made very few errors (92.5\% correct). Young and old rats' level of accuracy over the last five sessions of the training phase demonstrated that sufficient learning took place to justify assessment of memory loss.

The first analysis examined age differences on firstprobe accuracy rates during training and in the two delay 


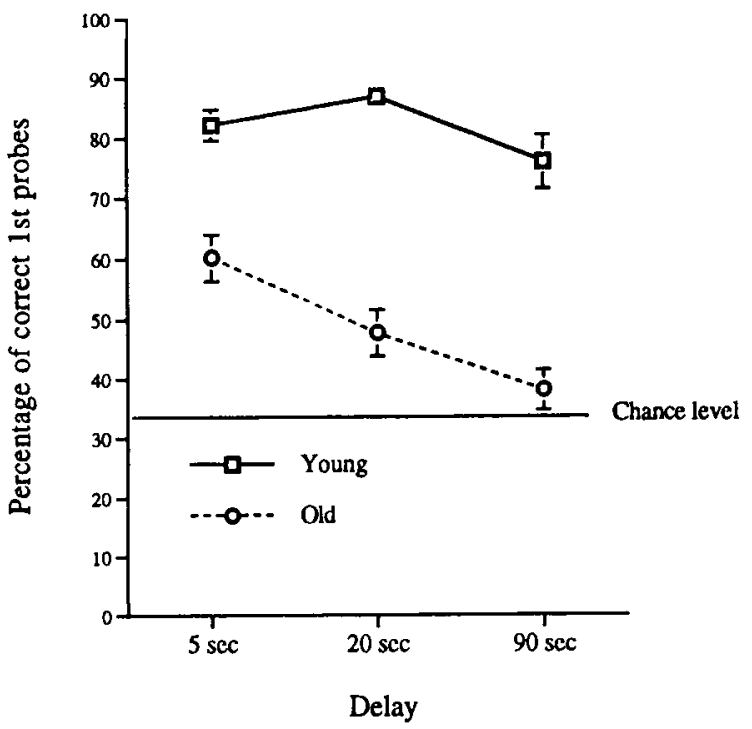

Figure 1. Percentage of correct first-probe responses by young and old groups as a function of the retention interval.

conditions (see Figure 1). An age $\times$ delay analysis of variance (ANOVA) for a mixed design showed that there were significant effects of age $[F(1,18)=1,355.85, p \leq$ $.0001]$ and delay $[F(2,36)=92.63, p \leq .0001]$. The age $\times$ delay interaction was also significant $[F(2,36)=$ $39.59, p \leq .0001]$. The significant interaction indicates that the rate of decline was not the same in each group. An analysis of simple main effects showed that there was a significant difference across delays for the young group $[F(2,36)=25.82, p \leq .01]$ as well as for the old group $[F(2,36)=106.4, p \leq .0001]$. Post hoc multiple comparison tests (Tukey, HSD, $p \leq .025$ ) revealed that young rats performed worse at the 90 -sec delay than at $3 \mathrm{sec}$ or $20 \mathrm{sec}$, but there was no significant difference between performance at the 3 - and the 20 -sec delays. The old rats' performance was significantly better at the 3 -sec delay than at both the 20 - and the 90-sec delays, and performance on the 20 -sec delay trials was also significantly better than that on the 90 -sec-delay trials. Taken together, these analyses indicate that memory for stimulus information was forgotten faster by the old rats.

Second-probe accuracy scores indicate whether firstprobe incorrect responses were caused by a failure to encode information about the sample arms or by fast memory decay. An age $\times$ delay ANOVA was performed to compare differences between young and old rats on second-probe accuracy (see Figure 2). The analysis revealed significant age $[F(1,18)=204.4, p \leq .0001]$ and delay $[F(2,36)=96.54, p \leq .0001]$ effects. The age $X$ delay interaction was also significant $[F(2,36)=51.5$, $p \leq .0001]$. Analysis of simple main effects revealed that there was a significant difference between the two age groups at all three retention intervals (all $F \mathrm{~s}>5.0, p \mathrm{~s} \leq$ .025 ). Young rats were significantly more accurate than old rats in the three delay conditions. Once again, the magnitude of the difference between young and old rats increased as a function of the delay.

The significant interaction also reveals that the rate of decline on second-probe performance was faster in the old group. Analysis of simple main effects across delays within each group showed that there was a significant difference between the three delays in the young group $[F(2,36)=33.95, p \leq .0001]$ as well as in the old group $[F(2,36)=114.05, p \leq .0001]$. Post hoc multiple comparisons test (Tukey, HSD, $p \leq .025$ ) indicated that young rats' accuracy was significantly higher on $3-\mathrm{sec}$ and 20 sec-delay trials than on 90 -sec-delay trials. There was no significant difference between the 3- and 20-sec-delay trials. In old rats however, performance on second probes was significantly higher on 3-sec-delay trials than on 20 and 90 -sec-delay trials, but there was no significant difference between the latter delays. In fact, old rats' performance at 20 and $90 \mathrm{sec}$ was at chance level on second probes, whereas young rats selected the correct arm reliably on second probes in the three delay conditions. The results on the short-delay trials pointed to an encoding deficit in the old rats. The analyses of the first and second probe run results for the delay condition indicate that, in addition, old rats suffered more rapid memory loss of information that was partially encoded.

Another way to look at the effect of decay with the present paradigm is to examine rats' selection of the first or second sample arms on their first-probe errors. If firstprobe errors were the result of a rapid memory decay, there should be faster forgetting of having entered the first sample arm in comparison with the second sample arm. Consequently, they should show a tendency to visit that arm more often.

For each group and each delay condition, the proportion of first-probe errors committed to the first sample

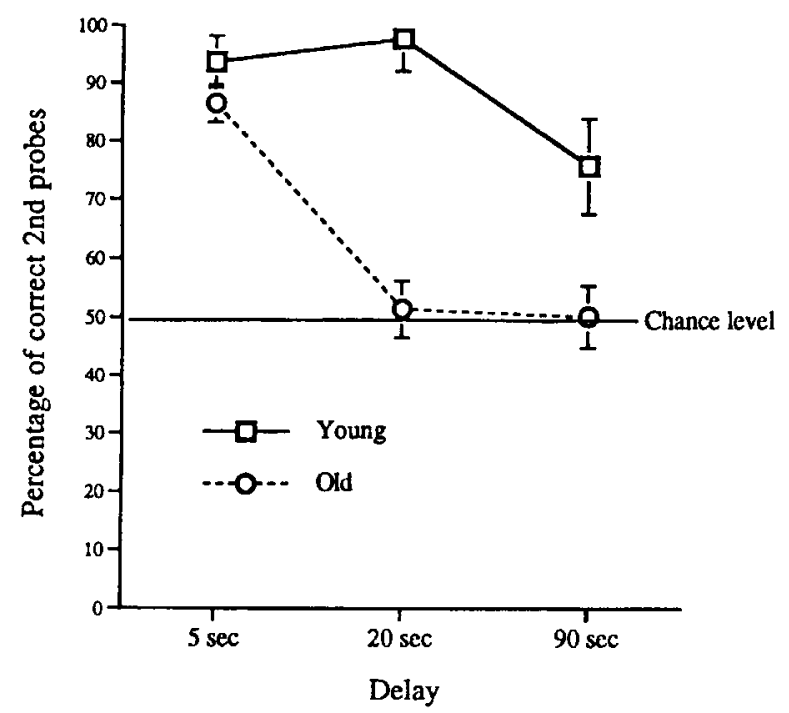

Figure 2. Percentage of correct seciond-probe responses by young and old groups as a function of the retention interval. 


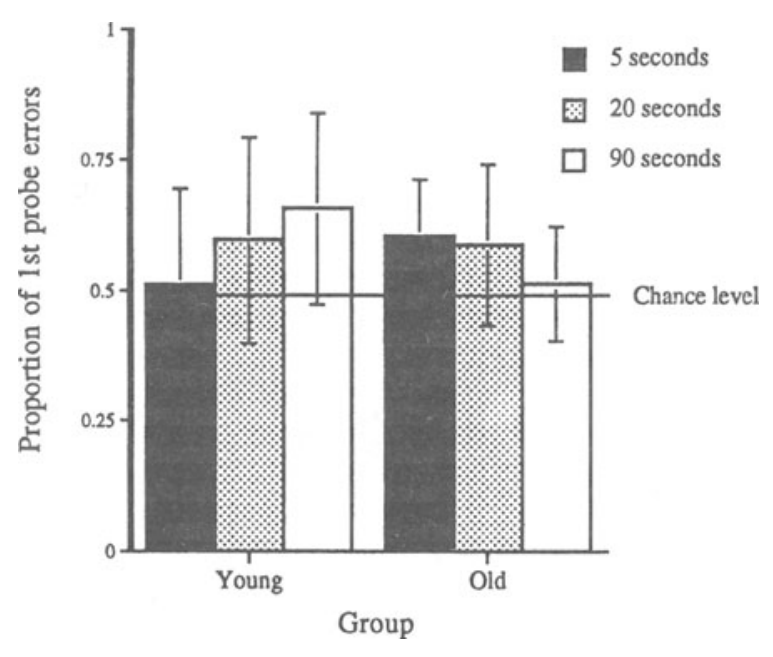

Figure 3. Proportion of first-probe errors made to the first sample arm in young and old groups as a function of the retention interval.

arm was compared with its occurrence by chance (.50) (see Figure 3). A one-sample $t$ test was computed for each group and each delay. The $t$ tests showed that young rats chose the first sample arm significantly more often than chance in the 90 -sec delay condition $[t(9)=2.7, p \leq$ $.01]$, but there were no significant arm preferences in the other two delay conditions. In contrast, old rats displayed a stronger tendency to visit the first sample arm on their first-probe errors on 3-sec-delay $[t(9)=1.55, p=.08]$ and 20-sec-delay $[t(9)=1.79, p=.053]$ trials. On 90 sec-delay trials, first-probe incorrect responses were distributed equally between the first and second sample arms, suggesting that there was a complete memory loss in old rats.

\section{DISCUSSION}

The present results clearly demonstrated that old rats were severely impaired, relative to young adult rats, in learning the NMTS task. Moreover, the age-related deficit increased with the length of time rats were required to retain trial-specific stimulus information before testing on the probe runs. The demonstration of age differences in NMTS learning as well as the time-dependent memory loss is consistent with previous reports involving similar MTS and NMTS paradigms (Dunnett et al., 1988; Dunnett et al., 1990; Pontecorvo et al., 1988; Walker et al., 1988; Winocur, 1986, 1992a, 1992b).

The present study represents a first attempt to analyze the behavior of old rats in a test of NMTS in order to assess more precisely the nature of their deficit. Previous studies, involving similar tasks, were not designed to evaluate age-related impairments or assess the possibility of an interaction between impaired learning and memory functions. The present results provide evidence that old rats suffer a decline in their ability to encode new information and that this encoding failure combines with a retention deficit to affect overall performance.
On typical tests of MTS or NMTS, where subjects must select stimuli that match or do not match sample stimuli, only one measure is obtained within each trial. On standard acquisition trials, this measure, typically a right or a wrong response, permits inferences about the animal's ability to learn the task. At longer retention intervals, it provides information about rate of memory loss. In neither case is it possible to be more precise with respect to specific processes affected in the aged animals.

The critical feature of the multiple probe NMTS task is that it provides a second measure within the same trial that enables analyses of performance failure. The combination of first and second probe runs can provide information about encoding ability and retention of encoded information, as well as interactions involving both processes. For example, when there is a very brief delay between study and probe runs, an error on first probe followed by a correct response on second probe would indicate impaired, but partial, encoding of the stimulus information on the study runs. At long delays, second-probe performance provides insight into whether first-probe errors resulted from a combined effect of encoding and memory loss.

In the present study, at the 3-sec delay, old rats were substantially less accurate than young rats on first probe. While this result points to an encoding deficit, it is conceivable that a failure to retrieve encoded information contributed to the deficit. It is also conceivable that, despite the very short study-probe interval, old rats failed to retain information about the sample stimuli. In fact, old and young rats performed extremely well on second probe runs. The most likely interpretation is that the old rats' relatively poor performance on the first probe runs was due to a partial encoding deficit.

There was no evidence to support the view that the old rats' impairment was related to retrieval failure. A retrieval deficit interpretation would predict equally poor performance on first and second probe runs and could not account for the dramatic improvement displayed by old rats on second probe runs at short delay. Old and young groups displayed about $90 \%$ accuracy on these runs. In general, age differences in retrieval are most commonly associated with tests of free recall rather than recognition tests, which normally provide sufficient cues to minimize demand on internal retrieval processes (Craik \& Jennings, 1992; Huppert, 1991). The NMTS task used here can be considered a test of recognition memory, in that during the probe runs, rats were required to choose among three response alternatives.

Although old rats showed evidence of substantial learning after 20 sessions, their performance was not as good as that of young rats before being submitted to delay trials. It could be argued that old rats' lower accuracy at the 3-sec delay resulted from poorer acquisition of the task and can be interpreted exclusively in terms of a learning deficit. There is certainly ample evidence that old rats suffer learning impairment on a variety of tasks (Aggleton et al., 1989; Dunnett et al., 1988; Winocur, 1992a, 1992b). Conceivably, the old rats' relatively poor performance on first-probe trials could be explained in 
this way, but such an interpretation on its own cannot account for this group's dramatic improvement on secondprobe trials. Our interpretation is that age differences in acquiring the NMTS rule are related directly to the animal's failure in encoding relevant information.

The finding that the old group improved significantly on second probe runs is also inconsistent with a retention deficit interpretation and strongly argues for a partial encoding deficit. On the other hand, there was some evidence that old rats experienced at least some memory loss even at the short delay. Examination of response patterns revealed that, when old rats made errors, they were more often choices to the arm that they had entered on the first study run than to the second study run. In contrast, first probe errors by young rats were equally distributed between the first and second sample runs. Consistent with a memory loss interpretation, this result indicates that, after a delay as short as $3 \mathrm{sec}$, old rats were more likely than young rats to forget that the first sample arm represented an incorrect choice on the probe test runs.

Further evidence of age-related memory loss was revealed by the analysis of first- and second-probe responses at 20- and 90-sec retention intervals. Old rats' first-probe accuracy declined steadily as the study-probe delays were increased. For this group, accuracy rates differed significantly at all delays. By comparison, in young rats, memory loss was observed only on 90 -secdelay trials. This is consistent with similar reports of faster forgetting rates in normal old rats (Dunnett et al., 1988, 1990; Winocur, 1992a, 1992b). As expected, old rats also displayed memory loss on second probe runs. In fact, on 20-sec-delay trials, old rats' second probe performance did net exceed chance level. As with firstprobe performance, a significant decline in accuracy was observed in the young group only after a $90-\mathrm{sec}$ delay.

Having established that old rats suffered failures of encoding and retention, one must still ask whether their time-dependent deficit resulted from a combined effect of both types of impairment. That is, was old rats' accuracy on probe runs affected by faster forgetting of poorly encoded stimulus information than of information that was effectively encoded? To provide evidence for such an interaction, it is necessary to show that poorly encoded information is more susceptible to decay over long intervals.

Evidence that poorly encoded information was vulnerable to rapid decay comes from a comparison of decline on first- and second-probe performance over the three retention intervals. If it is assumed that during encoding of stimulus information, some value is attached to each arm of the maze, it follows that, in efficient encoding, the greatest value with respect to selecting the correct arm on the probe runs is attached to the nonmatching arm, with less value to the other two arms. In contrast, poor or partial encoding would have the effect of attaching inappropriate values to the various arms. For example, if a rat failed to encode any information about the sample arms, equal value would be attached to all arms and selection on the probe runs would occur on a random basis. In a more typical case, involving partial encoding of sample stimulus information, the highest value would be attached to one of the wrong arms with the next highest value attached to the correct nonmatching arm. At the short delay, this outcome would be reflected in large numbers of first probe errors followed by improved accuracy on second probe runs, as was found to be the case. Because second-probe performance is based on partially encoded information, if encoding and retention deficits interact with each other, the rate of memory loss and performance decline should be faster on this measure than on first-probe correct responses, which are based on more efficient encoding of information. That this was precisely the pattern observed in old rats provides evidence that encoding and retention deficits interacted with each other and combined to affect NMTS performance of old rats, particularly when study and probe trials were distributed over relatively long delays.

Finally, from a neuropsychological perspective, the present results are consistent with reports that relate learning and memory deficits in old rats to deterioration of specific brain regions, most notably the prefrontal cortex (Boone, Miller, \& Lesser, 1993) and the hippocampus (Geinisman, deToledo-Morrell, Morrell, \& Heller, 1995). The latter structures are particularly susceptible to the aging process, and there is growing evidence from brain-imaging studies with humans that structure atrophy and dysfunctions in these regions correlate with impaired learning and memory performance (Albert \& Stafford, 1986). Behavioral studies involving young adult rats with localized lesions and normal aged rats suggest that learning deficits on conditional learning tasks similar to that used in the present study can result from damage to the prefrontal region (Winocur, 1992a, 1992b). In addition to implicating prefrontal dysfunction in the NMTS deficit of old rats, the present data suggest that this structure may contribute to the normal encoding of stimulus information (see also Kesner \& Holbrook, 1987; Poucet, 1990). Furthermore, the type of memory deficit displayed by the old rats is typical of that associated with hippocampal damage (Winocur, 1988) and is consistent with the view that this structure is critical for maintaining representations of newly formed associations between unrelated stimuli (Moscovitch, 1994; Winocur, 1988).

In summary, Roitblat's multiple probe technique (Adams-Pepper et al., 1992; Roitblat \& Harley, 1988; Roitblat et al., 1989) was used successfully to demonstrate age differences between young and old rats in learning an NMTS task. Old rats also showed faster forgetting of specific stimulus information as intervals between study and probe runs were increased. An important advantage of the multiple probe procedure was its ability to dissociate cognitive processes affected by normal aging and demonstrate their respective contributions to performance deficits on the NMTS task. Specifically, it was shown that age differences on the task could 
be attributed to the combined effect of encoding failure and rapid memory loss. Finally, the age-related performance deficits were linked to prefrontal and hippocampal dysfunctions, suggesting that the multiple probe procedure may be usefully applied to the neuropsychological study of memory disorders.

\section{REFERENCES}

Adams-Pepper, S., Gagnon, S., Fore, S., \& Roitblat, H. L. (1992). Rats remember not wisely but too well. Animal Learning \& Behavior, 20, 363-372.

Aggleton, J. P., Blindt, H. S., \& CANdy, J. M. (1989). Working memory in aged rats. Behavioral Neuroscience, 103, 975-983.

AlbERT, M. S., \& STAFFord, J. L. (1986). CT scan and neuropsychological relationship in aging and dementia. In G. Goldstein \& R. E. Taske (Eds.), Advances in clinical neuropsychology (Vol. 3, pp. 3153). New York: Plenum.

Boone, K. B., MiLler, P. L., \& Lesser, I. M. (1993). Frontal lobe cognitive functions in aging: Methodologic considerations. Dementia, 4, 232-236.

Craik, F. I. M., \& Jennings, J. M. (1992). Human memory. In F. I. M. Craik \& T. A. Salthouse (Eds.), The handbook of aging and cognition (pp. 51-110). Hillsdale, NJ: Erlbaum.

Dunnett, S. B., Evenden, J. L., \& Iversen, S. D. (1988). Delaydependent short-term memory deficits in aged rats. Psychopharma$\operatorname{colog} y, 96,174-180$.

Dunnett, S. B., Martel, F. L., \& Iversen, S. D. (1990). Proactive interference effects on short-term memory in rats: II. Effects in young and aged rats. Behavioral Neuroscience, 104, 666-670.

Gallagher, M., \& Pelleymounter, M. A. (1988). Spatial learning deficits in old rats: A model for memory decline in the aged. Neurobiology of Aging, 9, 549-556.

Geinisman, Y., DeToledo-Morrell, L., Morrell, F., \& Heller, R. E. (1995). Hippocampal markers of age-related memory dysfunction: Behavioral, electrophysiological and morphological perspectives. Progress in Neurobiology, 45, 223-252.

HuPPERT, F. A. (1991). Memory function in older adults: Remembering new information. In F. Boller \& J. Grafman (Eds.), Handbook of neuropsychology (pp. 123-147). Amsterdam: Elsevier.

KeSNER, R. P., \& Holbrook, T. (1987). Dissociation of item and order spatial memory in rats following medial prefrontal cortex lesions. Neuropsychologia, 25, 653-664.

Moscoviтch, M. (1994). Memory and working with memory: Evaluation of a component process model and comparisons with other models. In D. L. Schacter \& E. Tulving (Eds.), Memory systems 1994 (pp. 269-310). Cambridge, MA: MIT Press.

Pontecorvo, M. J., Clissold, D. B., \& ContI, L. H. (1988). Agerelated cognitive impairments as assessed with an automated repeated measures memory task: Implications for the possible role of acetylcholine and norepinephrine in memory dysfunction. Neurobiology of Aging, 9, 617-625.

PouCET, B. (1990). A further characterization of the spatial problemsolving deficit induced by lesions of the medial frontal cortex in the rat. Behavioural Brain Research, 41, 229-237.

RoITBLAT, H. L. (1980). Codes and coding processes in pigeon shortterm memory. Animal Learning \& Behavior, 8, 341-351.

Roitblat, H. L., \& Harley, H. E. (1988). Spatial delayed matchingto-sample performance by rats: Learning, memory, and proactive interference. Journal of Experimental Psychology: Animal Behavior Processes, 14, 71-82.

Roitblat, H. L., Harley, H. E., \& Helweg, D. A. (1989). The effects of scopolamine on proactive interference and spatial delayed matchingto-sample performance in rats. Psychobiology, 17, 402-408.

Walker, L. C., Kitt, C. A., Struble, R. G., Wagster, M. V., Price, D. L., \& CoRK, L. C. (1988). The neural basis of memory decline in aged monkeys. Neurobiology of Aging, 9, 657-666.

WinOcur, G. (1986). Memory decline in aged rats: A neuropsychological interpretation. Journal of Gerontology, 41, 758-763.

WinOcur, G. (1988). A neuropsychological analysis of memory loss with age. Neurobiology of Aging, 9, 487-494.

Winocur, G. (1992a). A comparison of normal old rats and young adult rats with lesions to the hippocampus or prefrontal cortex on a test of matching-to-sample. Neuropsychologia, 9, 769-781.

Winocur, G. (1992b). Conditional learning in aged rats: Evidence of hippocampal and prefrontal cortex impairment. Neurobiology of Aging, 13, 131-135.

Zornetzer, S. F., Thompson, R., \& Rogers, J. (1982). Rapid forgetting in aged rats. Behavioral \& Neural Biology, 36, 49-60.

(Manuscript received April 21, 1995; revision accepted for publication August 1, 1995.) 